\title{
Effect of prior antibiotic or chemotherapy treatment on immunotherapy response in non-small cell lung cancer
}

Andrew F. Nyein ${ }^{1}$, Shahla Bari², Stephanie Hogue ${ }^{1,3}$, Yayi Zhao ${ }^{1}$, Bradley Maller ${ }^{4}$, Sybil Sha ${ }^{5}$, Maria F. Gomez ${ }^{1}$, Dana E. Rollison ${ }^{1}$ and Lary A. Robinson ${ }^{1,3^{*}}$

\begin{abstract}
Background: Treatment outcomes of advanced non-small cell lung cancer (NSCLC) have substantially improved with immune checkpoint inhibitors (ICl), although only approximately $19 \%$ of patients respond to immunotherapy alone, increasing to $58 \%$ with the addition of chemotherapy. The gut microbiome has been recognized as a modulator of $\mathrm{ICl}$ response via its priming effect on the host immune response. Antibiotics as well as chemotherapy reduce gut microbial diversity, hence altering composition and function of the gut microbiome. Since the gut microbiome may modify $\mathrm{ICl}$ efficacy, we conducted a retrospective study evaluating the effects of prior antibiotic or chemotherapy use on NSCLC patient response to ICl.
\end{abstract}

Methods: We retrospectively evaluated 256 NSCLC patients treated between 2011-2017 at Moffitt Cancer Center with $\mid \mathrm{Cl} \pm$ chemotherapy, examining the associations between prior antibiotic or chemotherapy use, overall response rate and survival. Relative risk regression using a log-link with combinatorial expectation maximization algorithm was performed to analyze differences in response between patients treated with antibiotics or chemotherapy versus patients who didn't receive antibiotics or chemotherapy. Cox proportional hazards models were constructed to evaluate associations between risk factors and overall survival.

Results: Only 46 (18\% of 256) patients used antibiotics prior to and/or during ICI treatment, and 146 (57\%) had prior chemotherapy. Antibiotic users were $8 \%$ more likely to have worse overall response rate (RR:1.08; Cl:0.93-1.26; $p=0.321$ ), as well as a 35\% worse overall survival (HR:1.35; Cl:0.91-2.02; $p=0.145$ ), although results were not statistically significant. However, prior use of chemotherapy was significantly associated with poor ICI response (RR:1.24; Cl:1.05-1.47; $p=0.013$ ) and worse overall survival (HR:1.47; Cl:1.07-2.03; $p=0.018)$.

Conclusions: Patients receiving antibiotics prior to and/or during $\mathrm{ICl}$ therapy might experience worse treatment outcomes and survival than unexposed patients, although these associations were not statistically significant and hence warrant further prospective study. Prior chemotherapy significantly reduced ICl response and overall survival. Antibiotic or chemotherapy exposure may negatively impact ICI response, perhaps through disruption of the eubiotic gut microbiome.

Keywords: Non-small cell lung cancer, Immune checkpoint inhibitors, Immunotherapy, Antibiotics, Chemotherapy, Microbiome

\footnotetext{
*Correspondence: lary.robinson@moffitt.org

${ }^{1}$ Department of Cancer Epidemiology, Moffitt Cancer Center, Tampa, FL

33612, USA

Full list of author information is available at the end of the article
} 


\section{Background}

Lung cancer represents $13 \%$ of all newly diagnosed cancers and is the leading cause of cancer-related death, with an overall 5 -year survival rate of only $15 \%[1,2]$. Of the various histological subtypes, non-small cell lung cancer (NSCLC) represents the majority of lung cancers (80$85 \%$ ), with treatment options ranging from radiation and chemotherapy to surgical resection $[1,3]$. Although treatment of early-stage disease (stages IA to IIB) results in a 5-year survival rate of 49-83\% [4], early detection can be challenging due to lack of biomarkers and a prolonged latency period $[5,6]$. Nearly $50 \%$ of lung cancers are therefore diagnosed at advanced clinical stage [7], where the cure rate is markedly reduced $[8,9]$.

Immune checkpoint inhibitors (ICIs) have changed the treatment paradigm of NSCLC, markedly improving response rates and survival [10]. ICIs function by blocking important regulatory receptors, thus releasing the brake on immune cells and allowing immune-mediated tumor recognition and killing [11, 12]. Unfortunately, over $50 \%$ of patients may fail to respond to ICI-chemotherapy combination treatment [10]. Those who respond, however, may experience a response duration upwards of 3 years [13]. Identifying biomarkers and modulators of ICI response is therefore critically important to distinguish those who might receive treatment benefit from those who might not.

Recently, the gut microbiome has been studied as a potential mediator of ICI efficacy. The influence of the gut microbiota on ICI response has been established by fecal microbiota transplant (FMT), whereby FMT from responding patients improved ICI response when transplanted to germ-free mice compared to mice transplanted with non-responding patient stool [14]. In humans, recent studies in various cancers, including NSCLC, have identified higher microbial diversity and enrichment of Bifidobacterium [15], Akkermansia [16], Faecalibacterium [14], and well-known short-chain fatty acid-producing bacteria [17] in ICI responders versus non-responders. Given recent evidence for the potential role of the gut microbiome in mediating ICI response, it follows that medications, including antibiotics (ATBs) and cytotoxic chemotherapy, that can alter the gut microbiome and lead to dysbiosis may impact ICI response. Indeed, recent evidence shows that when cancer patients receive ICI therapy and have been exposed to ATBs prior to treatment, they experience worse survival [18-21].

While numerous studies have evaluated the role of prior and concurrent ATBs on ICI response, results have been inconsistent, with conflicting reports ranging from no effect [22] to an unfavorable effect on progression free survival and/or overall survival (OS) [23]. Furthermore, most studies have evaluated only the effect of ATBs on ICI response, whereas the effect of pretreatment chemotherapy on ICI response has been overlooked. To explore the role of the gut microbiome in mediating ICI response, we conducted a retrospective study to evaluate the effects of pre-treatment and concurrent ATB as well as cytotoxic chemotherapy exposure on ICI response in patients with advanced NSCLC (stage III/IV).

\section{Methods \\ Study population}

Of the 381 patient medical records screened, 256 were deemed eligible with adequate follow-up data. Eligible patients included men and women $\geq 18$ years of age diagnosed with primary stage III or IV NSCLC, adenocarcinoma or squamous cell carcinoma, treated with ICIs at Moffitt Cancer Center (MCC) between January 1, 2011 and March 31, 2017. ICI agents included anti-PD-1, antiPD-L1, or anti-CTLA-4, alone or in combination with other agents such as targeted therapy or chemotherapy. Approval for this retrospective chart review was obtained from the MCC Scientific Review Committee and Chesapeake Institutional Review Board, Protocol MCC 19,162.

\section{Clinical data abstraction}

Clinical data was abstracted and transcribed into a secure database. Data elements were defined according to standard definitions and finalized in consultation with clinical investigators. Second-level abstractions were performed on a random sample of $20 \%$ of the medical records to ensure an accurate and unbiased abstraction process. Discrepancies were discussed and resolved by consensus of the research team.

Recent ATB use was assessed for the period two months before and up to one month after the initial ICI dose, and patients were dichotomized as having received or not received ATBs in this three-month period around ICI initiation. The primary clinical outcome was clinician-assessed response [complete response (CR), partial response (PR), stable disease $(\mathrm{SD})$, or progressive disease (PD)] using radiographic interpretations with the Response Evaluation Criteria in Solid Tumors (RECIST) version 1.1 [24]. The best clinical response recorded within six months of ICI start was abstracted and patients were categorized as responders (CR/PR) or non-responders (SD/PD). OS, defined as the time between start of ICI and death or censored at date of last follow-up, was examined as a secondary clinical outcome.

Sociodemographic characteristics such as sex, age, race/ethnicity, smoking status, as well as date of death or last contact were recorded. Indication for ATB use, duration, dose and type of ATB used were also recorded. 
Table 1 Baseline characteristics by antibiotic exposure

\begin{tabular}{|c|c|c|c|c|c|c|c|}
\hline \multirow[b]{2}{*}{ Factors } & \multirow[b]{2}{*}{ Total $(n=256)$} & \multicolumn{3}{|l|}{ Exposure } & \multicolumn{3}{|l|}{ Outcome } \\
\hline & & ATB (-) Group $(n=210)$ & $\begin{array}{l}\text { ATB }(+) \\
\text { Group } \\
(n=46)\end{array}$ & $P$-value & CR \& PR $(n=71)$ & SD \& PD $(n=185)$ & $P$-value \\
\hline Age (Years) Mean [SD] & $65.5[9.5]$ & $65.5[9.6]$ & $65.3[9.4]$ & 0.942 & $66.5[9.7]$ & $65.1[9.4]$ & 0.400 \\
\hline \multicolumn{8}{|l|}{ Ethnicity- No. (\%) } \\
\hline Non-Hispanic & $243(94.9)$ & $200(95.2) 10$ & $43(93.5) 3$ & 0.903 & $69(97.2) 2$ & $174(94.1)$ & \\
\hline Hispanic/Latino & $13(5.1)$ & $(4.8)$ & $(6.5)$ & & $(2.8)$ & $11(5.9)$ & 0.482 \\
\hline \multicolumn{8}{|l|}{ Pack Years } \\
\hline Mean [SD] & $32.9[25.9]$ & $33.8[26.3]$ & $29.2[23.9]$ & 0.435 & $30.5[23.9]$ & $33.8[26.6]$ & 0.496 \\
\hline \multicolumn{8}{|l|}{ Clinical Stage- No. (\%) } \\
\hline Stage 3 & $16(6.2)$ & $14(6.7)$ & $2(4.3)$ & 0.801 & $5(7.0)$ & $11(5.9)$ & 0.971 \\
\hline Stage 4 & $240(93.8)$ & $196(93.3)$ & $44(95.7)$ & & $66(93.0)$ & $174(94.1)$ & \\
\hline \multicolumn{8}{|l|}{ Race- No. (\%) } \\
\hline White & $241(94.2)$ & $200(95.2)$ & $41(89.1)$ & & $65(91.5)$ & $176(95.1)$ & \\
\hline Black or African American & $9(3.5)$ & $7(3.3)$ & $2(4.3)$ & 0.109 & $3(4.2)$ & $6(3.2)$ & 0.429 \\
\hline Asian & $6(2.3)$ & $3(1.4)$ & $3(6.5)$ & & $3(4.2)$ & $3(1.6)$ & \\
\hline \multicolumn{8}{|l|}{ Gender- No. (\%) } \\
\hline Female & $120(47.0)$ & $98(46.7)$ & $22(47.8)$ & 1.000 & $29(40.8)$ & $91(49.2) 94$ & 0.290 \\
\hline Male & $136(53.0)$ & $112(53.3)$ & $24(52.2)$ & & $42(59.2)$ & $(50.8)$ & \\
\hline \multicolumn{8}{|l|}{ Smoking Status- No. (\%) } \\
\hline Never & $38(14.8)$ & $29(13.8)$ & $9(19.6)$ & & $10(14.1)$ & $28(15.1)$ & \\
\hline Former & $184(71.9)$ & $153(72.9)$ & $31(67.4)$ & 0.606 & $51(71.8)$ & $133(71.9)$ & 0.958 \\
\hline Current & $34(13.3)$ & $28(13.3)$ & $6(13.0)$ & & $10(14.1)$ & $24(13.0)$ & \\
\hline \multicolumn{8}{|l|}{ Marital Status- No. (\%) } \\
\hline Single & $23(9.0)$ & $19(9.0)$ & $4(8.7)$ & & $10(14.1)$ & $13(7.0)$ & \\
\hline Married/Cohabitating & $156(60.9)$ & $133(63.3)$ & $23(50.0)$ & 0.601 & $40(56.3)$ & $116(62.7)$ & 0.185 \\
\hline $\begin{array}{l}\text { Widowed/Divorced/Sepa- } \\
\text { rated }\end{array}$ & $48(18.8)$ & $38(18.1)$ & $10(21.7)$ & & $12(16.9)$ & $36(19.5)$ & \\
\hline Not Reported & $29(11.3)$ & $20(9.6)$ & $9(19.6)$ & & $9(12.7)$ & $20(10.8)$ & \\
\hline \multicolumn{8}{|l|}{ Prior Surgery- No. (\%) } \\
\hline No & $203(79.3)$ & $162(77.1)$ & $41(89.1)$ & 0.106 & $59(83.1)$ & $144(77.8)$ & 0.449 \\
\hline Yes & $53(20.7)$ & $48(22.9)$ & $5(10.9)$ & & $12(16.9)$ & $41(22.2)$ & \\
\hline \multicolumn{8}{|l|}{ Prior Chemotherapy- No. (\%) } \\
\hline No & $110(43.0)$ & $92(43.8)$ & $18(39.1)$ & 0.677 & $41(57.7)$ & $69(37.3)$ & $0.005^{*}$ \\
\hline Yes & $146(57.0)$ & $118(56.2)$ & $28(60.9)$ & & $30(42.3)$ & $116(62.7)$ & \\
\hline \multicolumn{8}{|l|}{ Prior Radiation- No. (\%) } \\
\hline No & $178(69.5)$ & $148(70.5)$ & $30(65.2)$ & 0.600 & $50(70.4)$ & $128(69.2)$ & 0.968 \\
\hline Yes & $78(30.5)$ & $62(29.5)$ & $16(34.8)$ & & $21(29.6)$ & $57(30.8)$ & \\
\hline \multicolumn{8}{|l|}{ Prior Targeted Therapy- №. (\%) } \\
\hline No & $215(84.0)$ & $173(82.4)$ & $42(91.3)$ & 0.203 & $64(90.1)$ & $151(81.6)$ & 0.141 \\
\hline Yes & $41(16.0)$ & $37(17.6)$ & $4(8.7)$ & & $7(9.9)$ & $34(18.4)$ & \\
\hline \multicolumn{8}{|l|}{ Histology- No. (\%) } \\
\hline Adenocarcinoma & $147(57.4)$ & $119(56.7)$ & $28(60.9)$ & & $43(60.6)$ & $104(56.2)$ & \\
\hline Squamous Cell Carcinoma & $59(23.0)$ & $49(23.3)$ & $10(21.7)$ & 0.865 & $15(21.1)$ & $44(23.8)$ & 0.818 \\
\hline Other NSCLC & $50(19.6)$ & $42(20.0)$ & $8(17.4)$ & & $13(18.3)$ & $37(20.0)$ & \\
\hline \multicolumn{8}{|l|}{ NSAID Use- No. (\%) } \\
\hline No & $235(91.8)$ & $190(90.5)$ & $45(97.8)$ & 0.177 & $63(88.7)$ & $172(93.0)$ & 0.394 \\
\hline Yes & $21(8.2)$ & $20(9.5)$ & $1(2.2)$ & & $8(11.3)$ & $13(7.0)$ & \\
\hline \multicolumn{8}{|l|}{ PPI Use- No. (\%) } \\
\hline No & $195(76.2)$ & $153(72.9)$ & $42(91.3)$ & $0.014^{*}$ & $50(70.4)$ & $145(78.4)$ & 0.241 \\
\hline
\end{tabular}


Table 1 (continued)

\begin{tabular}{|c|c|c|c|c|c|c|c|}
\hline \multirow[b]{2}{*}{ Factors } & \multirow[b]{2}{*}{ Total $(n=256)$} & \multicolumn{3}{|l|}{ Exposure } & \multicolumn{3}{|l|}{ Outcome } \\
\hline & & ATB (-) Group $(n=210)$ & $\begin{array}{l}\text { ATB }(+) \\
\text { Group } \\
(n=46)\end{array}$ & $P$-value & CR \& PR $(n=71)$ & SD \& PD $(n=185)$ & $P$-value \\
\hline Yes & $61(23.8)$ & $57(27.1)$ & $4(8.7)$ & & $21(29.6)$ & $40(21.6)$ & \\
\hline \multicolumn{8}{|l|}{ ECOG- No. (\%) } \\
\hline 0 & $57(22.3)$ & $50(23.8)$ & $7(15.2)$ & & $18(25.4)$ & $39(21.1)$ & \\
\hline 1 & $192(75.0)$ & $153(72.9)$ & $39(84.8)$ & 0.195 & $52(73.2)$ & $140(75.7)$ & 0.662 \\
\hline 2 & $6(2.3)$ & $6(2.9)$ & $0(0.0)$ & & $1(1.4)$ & $5(2.7)$ & \\
\hline Not Reported & $1(0.4)$ & $1(0.4)$ & $0(0.0)$ & & $0(0.0)$ & $1(0.5)$ & \\
\hline \multicolumn{8}{|c|}{ Charlson Comorbidity Index } \\
\hline Mean [SD] & $6.9[1.6]$ & $6.9[1.6]$ & $6.9[1.5]$ & 0.766 & $7.1[1.7]$ & $6.9[1.5]$ & 0.528 \\
\hline
\end{tabular}

Table 2 Reasons for ATB use, by treatment outcome

\begin{tabular}{|c|c|c|c|c|c|}
\hline & \multicolumn{3}{|c|}{$\begin{array}{l}\text { Treatment response within } \\
6 \text { months }\end{array}$} & \multirow{2}{*}{$\begin{array}{l}\begin{array}{l}\text { Duration } \\
\text { (days) }^{2}\end{array} \\
\text { Mean [SD] }\end{array}$} & \multirow{2}{*}{$\begin{array}{l}\text { Dose }(\mathrm{mg})^{2} \\
\text { Mean [SD] }\end{array}$} \\
\hline & $\begin{array}{l}\text { CR/PR } \\
(n=12)^{1}\end{array}$ & $\begin{array}{l}\text { SD/PD } \\
(n=34)\end{array}$ & $P$-value & & \\
\hline \multicolumn{6}{|c|}{ Surgery } \\
\hline No & 8 & 23 & 1.000 & 1.12 [0.33] & $1298.44(657.04)$ \\
\hline Yes & 4 & 11 & & & \\
\hline \multicolumn{6}{|l|}{ RTI } \\
\hline No & 5 & 16 & 1.000 & 7.84 [6.43] & $587.07(590.89)$ \\
\hline Yes & 7 & 18 & & & \\
\hline \multicolumn{6}{|c|}{ Other Reasons } \\
\hline No & 7 & 20 & 1.000 & $13.65[10.60]$ & 375.24 (265.38) \\
\hline Yes & 5 & 14 & & & \\
\hline
\end{tabular}

${ }^{*}$ Statistically Significant for Fisher's exact test, when $p<0.05$; 1: no CR among ATB users, only PR. 2: this is measured at the level of each ATB use, not at the individual patient-level. $C R$ Complete response, $P R$ Partial response, $P D$ Progressive disease, $S D$ Stable disease, $R T I$ Respiratory tract infection, including bronchitis, pneumonia, upper respiratory tract infection, and sinusitis. Other reasons include patients taking antibiotics for acne, biopsy, cholangitis, colitis, ear cellulitis, leukocytosis, metastasis, rash, urinary tract infection, prophylaxis. Duration of antibiotic use for each reason was not conducted at patient-level, but instead calculated with antibiotic-use level analysis. Each patient may have overlapping antibiotic use; each use was treated as a single observation

Furthermore, prior chemotherapy within 6 months of ICI treatment, other medication usage, and comorbidities [evaluated and graded according to the Charlson Comorbidity Index $(\mathrm{CCI})]$ were abstracted from the medical record.

\section{Statistical analyses}

Baseline characteristics were reported and stratified by ATB use (primary exposure) and by clinician-assessed response (primary outcome). Wilcoxon rank-sum test and Chi-squared test were used to assess whether baseline characteristics were associated with the exposure and/or outcome. Treatment response was cross-tabulated with reason for ATB use, with p-values calculated using Fisher's exact test. Mean and standard deviation of the duration and dose of ATB use were also reported.

Relative risk (RR) regression using a log-link with combinatorial expectation maximization (CEM) algorithm [25] was used to assess the association between recent ATB use and other risk factors with clinical response. In addition to univariate RR models, a multivariate model (multivariate 1) was constructed to assess the association between ATB use and clinical response, adjusting for baseline characteristics that were found to be associated with either exposure or outcome. Subsequently, a more inclusive model (multivariate 2) was constructed to include additional relevant factors, such as age, ECOG, and prior targeted therapy. Kaplan-Meier analyses were performed, and Cox proportional hazard models were constructed to examine the association between risk factors and OS. Adjusted Cox models incorporated relevant clinical variables, such as age, prior chemotherapy and ECOG performance status, determined by recursive stepwise selection. Statistical analyses were performed using R, version 4.0.4 (R Foundation for Statistical Computing, Vienna, Austria, 2019). P values $<0.05$ were considered statistically significant.

\section{Results}

\section{Patient characteristics}

This retrospective analysis included 256 NSCLC patients. Mean patient age was 65 years (range $45-85$ years), with a relatively equal number of men and women (136 men, $53 \%)$. The majority were white $(241,94 \%)$, married (156, $61 \%)$, and former smokers (184, 72\%). Most (198, 77\%) 
of these patients had ECOG performance status of 1-2 and $94 \%$ (240) were diagnosed with stage 4 disease. Fifty seven percent of patients (147) had adenocarcinoma, $23 \%$ (53) had squamous cell carcinoma and 20\% (50) had other subtypes of NSCLC. Over half of patients (146, $57 \%)$ had received chemotherapy within six months of ICI treatment, 31\% received radiation treatment and 16\% received other targeted treatments prior to ICI therapy. Complete demographic and clinical data are shown in Table 1. Overall, there were no significant differences in baseline demographic and clinical patient characteristics between ATB users and non-users, except for the use of proton pump inhibitors (PPIs), which was significantly more common in patients unexposed to ATBs versus those who were exposed.

\section{Antibiotic use}

Of the total 256 patients, 46 (18\%) received ATBs within 60 days of ICI initiation or concurrently with the first month of ICI therapy. Both oral and intravenous ATBs were used, including $\beta$-lactams, fluoroquinolones, macrolides, cephalosporins and tetracyclines. The three most prescribed ATBs were levofloxacin $(n=15)$, cefazolin $(n=14)$, and azithromycin $(n=8)$. ATBs were prescribed to patients for various indications, with the most frequent being prophylactic use prior to surgery $(n=15)$ and treatment of upper respiratory tract infections $(n=25)$, as shown in Table 2. The average duration of ATB use was approximately 1 day for surgical prophylaxis and 8 days for respiratory tract infection.

\section{Treatment response}

At 6 months following ICI start, 71 patients $(28 \%)$ were considered ICI responders (CR/PR). The disease control rate was about two-thirds (66\%), that is, 168 patients achieved a response of either CR, PR, or SD at 6 months post-ICI start. After adjusting for PPI use, prior chemotherapy, age, ECOG performance status and prior targeted therapy, relative risk analyses revealed that ATB-treated patients were $8 \%$ more likely to be ICI non-responders (SD/PD) compared to ATB-untreated patients (Table 3). However, this association did not reach statistical significance (RR: 1.08; CI:0.93-1.26; $p=0.321$ ). At 12 months of follow up, results were not significantly different (data not shown). Additionally, there were no significant differences in treatment response based on the reasons for antibiotic use (Table 2).

Interestingly, prior exposure to chemotherapy was significantly associated with an unfavorable ICI response

Table 3 Relative risk of poor clinical response

\begin{tabular}{|c|c|c|c|c|c|c|c|}
\hline & \multicolumn{2}{|c|}{ Best Response in 6 months } & \multirow{3}{*}{$\begin{array}{l}\text { Univariate } \\
\text { Relative Risk (95\% Cl) }\end{array}$} & \multirow{2}{*}{\multicolumn{2}{|c|}{ Multivariate $^{1}$}} & \multirow{2}{*}{\multicolumn{2}{|c|}{ Multivariate $^{2}$}} \\
\hline & \multirow{2}{*}{$\begin{array}{l}\text { CR/PR }(n=71) \\
N(\%)\end{array}$} & \multirow{2}{*}{$\begin{array}{l}\text { SD/PD }(n=185) \\
N(\%)\end{array}$} & & & & & \\
\hline & & & & Relative Risk (95\% Cl) & $P$-value & Relative Risk $(95 \% \mathrm{CI})$ & $P$-value \\
\hline \multicolumn{8}{|c|}{ Antibiotic Use } \\
\hline ATB- & $59(28.1)$ & $151(71.9)$ & 1.00 & 1.00 & & 1.00 & \\
\hline ATB + & $12(26.1)$ & $34(73.9)$ & $1.03(0.85-1.24)$ & $1.04(0.88-1.24)$ & 0.633 & $1.08(0.93-1.26)$ & 0.321 \\
\hline \multicolumn{8}{|l|}{ PPI Use } \\
\hline No & $50(25.6)$ & $145(74.4)$ & 1.00 & 1.00 & & 1.00 & \\
\hline Yes & $21(34.4)$ & $40(65.6)$ & $1.00(0.82-1.22)$ & $1.00(0.83-1.21)$ & 1.000 & $1.00(0.82-1.21)$ & 1.000 \\
\hline \multicolumn{8}{|c|}{ Prior Chemotherapy } \\
\hline No & $41(37.3)$ & $69(62.7)$ & 1.00 & 1.00 & & 1.00 & \\
\hline Yes & $30(20.5)$ & $116(79.5)$ & $1.27(1.07-1.50)$ & $1.26(1.07-1.48)$ & $0.007^{*}$ & $1.24(1.05-1.47)$ & $0.013^{*}$ \\
\hline \multicolumn{8}{|c|}{ Age (binary) } \\
\hline$<=67$ & $39(27.5)$ & $103(72.5)$ & 1.00 & NA & NA & 1.00 & \\
\hline$>67$ & $32(28.1)$ & $82(71.9)$ & $1.00(0.86,1.17)$ & NA & NA & $1.00(0.87-1.15)$ & 1.000 \\
\hline \multicolumn{8}{|c|}{ ECOG Performance Status } \\
\hline 0 & 18 (31.6) & $39(68.4)$ & 1.00 & NA & NA & 1.00 & \\
\hline $1 / 2$ & $53(26.8)$ & $145(73.2)$ & $1.07(0.88-1.30)$ & NA & NA & $1.06(0.89-1.26)$ & 0.530 \\
\hline \multicolumn{8}{|c|}{ Targeted Therapy } \\
\hline No & 64 (29.8) & $151(70.2)$ & 1.00 & NA & NA & 1.00 & \\
\hline Yes & $7(17.1)$ & 34 (82.9) & $1.18(1.00-1.39)$ & NA & NA & $1.11(0.95-1.29)$ & 0.188 \\
\hline
\end{tabular}

"Statistically Significant, when $p<0.05$; 1: Adjusted for significant risk factors in Table 1/adjusted for PPI and Chemotherapy; 2: Adjusted for PPI, Chemotherapy, Age (cut at the median age of 67), ECOG (0 vs. combined 1/2), and Targeted Therapy; ${ }^{*}$ Negative response ( SD\&PD) is considered as event/1. Positive response (CR\&PR) is considered as reference/ $0 . C R$ complete response, $P P$ Proton pump inhibitor, $P R$ partial response, $P D$ progressive disease, $S D$ Stable disease 
Table 4 Cox proportional hazards model for overall survival

\begin{tabular}{|c|c|c|c|c|c|}
\hline & \multirow{2}{*}{$\begin{array}{l}\text { Alive }(n=84) \\
N(\%)\end{array}$} & \multirow{2}{*}{$\begin{array}{l}\text { Deceased }(n=172) \\
N(\%)\end{array}$} & \multirow{2}{*}{$\begin{array}{l}\text { Univariate } \\
\text { Hazard Ratio } \\
(95 \% \mathrm{Cl})\end{array}$} & \multicolumn{2}{|l|}{ Multivariate $^{1}$} \\
\hline & & & & Hazard Ratio $(95 \% \mathrm{Cl})$ & $P$-value \\
\hline \multicolumn{6}{|c|}{ Antibiotic Use } \\
\hline ABT- & $70(83.3)$ & $140(81.4)$ & 1.00 & 1.00 & \\
\hline $\mathrm{ABT}+$ & $14(16.7)$ & $32(18.6)$ & $1.33(0.91-1.96)$ & $1.35(0.91-2.02)$ & 0.140 \\
\hline \multicolumn{6}{|l|}{ PPI Use } \\
\hline No & $62(73.8)$ & $133(77.3)$ & 1.00 & 1.00 & \\
\hline Yes & $22(26.2)$ & $39(22.7)$ & $0.91(0.63-1.29)$ & $0.92(0.64-1.33)$ & 0.667 \\
\hline \multicolumn{6}{|c|}{ Prior Chemotherapy } \\
\hline No & $44(52.4)$ & $66(38.4)$ & 1.00 & 1.00 & \\
\hline Yes & $40(47.6)$ & $106(61.6)$ & $1.42(1.04-1.93)$ & $1.47(1.07-2.03)$ & $0.018^{*}$ \\
\hline \multicolumn{6}{|c|}{ Age (mean [sd]) } \\
\hline NA & $65.6[10.1]$ & $65.4[9.2]$ & $1.00(0.99-1.02)$ & $1.00(0.98-1.02)$ & 0.933 \\
\hline \multicolumn{6}{|c|}{ ECOG Performance Status } \\
\hline 0 & $24(28.6)$ & $33(19.3)$ & 1.00 & 1.00 & \\
\hline $1 / 2$ & $60(71.4)$ & $138(80.7)$ & $1.75(1.19-2.57)$ & $1.75(1.19-2.57)$ & $0.005^{*}$ \\
\hline \multicolumn{6}{|c|}{ Targeted Therapy } \\
\hline No & $73(86.9)$ & $142(82.6)$ & 1.00 & 1.00 & \\
\hline Yes & $11(13.1)$ & $30(17.4)$ & $1.02(0.69-1.52)$ & $1.00(0.66-1.52)$ & 0.995 \\
\hline \multicolumn{6}{|c|}{ Smoking Status } \\
\hline Never & $13(15.5)$ & $25(14.5)$ & 1.00 & 1.00 & \\
\hline Former & $57(67.9)$ & $127(73.8)$ & $1.30(0.85-2.00)$ & $1.36(0.88-2.10)$ & 0.168 \\
\hline Current & $14(16.7)$ & 20 (11.6) & $1.02(0.57-1.84)$ & $1.06(0.58-1.92)$ & 0.859 \\
\hline
\end{tabular}

" Statistically Significant, when $p<0.05$; 1: Adjusted for PPI, Chemotherapy, Age (Continuous), ECOG (0 vs. combined 1/2), Targeted Therapy, and Smoking Status; *Dead considered as event/1. Alive is reference/0. PPI: Proton pump inhibitor

(SD/PD) (RR: 1.24; CI: 1.05-1.47; $p=0.013$ ) (Tables 1 and 3). However, the use of PPIs had no impact on treatment response at 6 months (Tables 1 and 3) or at 12 months (data not shown).

\section{Overall survival}

After adjusting the Cox proportional hazard model to account for potential confounding variables, including PPI use, prior treatment received, performance status and other factors, ATB use was associated with worse OS, however this was not statistically significant (HR:1.35;CI:0.91-2.02; $p=0.140$ ) (Table 4). The Kaplan-Meier survival analysis further corroborated this finding, as the median OS was consistently higher among ATB-untreated versus ATB-treated patients, though the log-rank p-value was not statistically significant (Fig. 1). However, prior chemotherapy use (HR:1.47; CI:1.07-2.03; $p=0.018$ ) and worse ECOG performance status (>0) (HR: 1.75 ; CI: $1.19-$ 2.57; $p=0.005)$ were associated with significantly worse OS.

\section{Discussion}

We investigated the effect of prior and concurrent ATB use on ICI response and OS, specifically for anti-PD-1/ anti-PDL-1 and anti-CTLA-4 immunotherapy treatment. Since stool samples were unavailable, ATB exposure was used as a proxy for gut microbial dysbiosis in these patients. After accounting for various confounders, ATB exposure was not statistically significantly associated with ICI treatment outcomes, although the trend suggested there may be an increased risk of poor outcomes in ATB users versus non-users. Prior chemotherapy, however, was significantly associated with worse response and overall survival. These results may suggest a potential role of medication-induced gut microbial dysbiosis in modulating immunotherapy response, which has been supported by a growing body of literature [20, 23, 26]. Specifically, the trillions of microorganisms that inhabit the gut play a critical role in priming our innate and adaptive immune response, including $\mathrm{CD} 4+/ \mathrm{CD} 8+\mathrm{T}$ cells, which are vital for ICI response [11]. Dysbiosis, which includes altered 
diversity as well as an imbalance in the gut microbial community that might be induced by different medications, may lead to altered or ineffective CD4+I CD8 + priming and, consequently, poor response to ICI [12].

Recent studies provide evidence that the effectiveness of anti-PD-L1 and anti-CTLA-4 blockade depends on the diversity of microbes as well as the presence of commensal Bifidobacterium and Bacteroides species, respectively $[27,28]$. Investigators have recently demonstrated that replenishing the gut microbiome with Akkermansia muciniphila in germ-free mice receiving FMTs from patients responding to immunotherapy promotes CCR9+CXCR3 + CD $4+$ T-lymphocyte migration into tumor beds [16]. These studies emphasize the importance of communication between the gut microbiota and the host's immune system in mediating immunotherapeutic effects. Further mechanistic studies are needed to clarify the potential effects of ATBs on gut microbial dysbiosis in the context of ICI therapy.

This study also surprisingly demonstrated that prior chemotherapy predicted poor outcomes, with a $47 \%$ increased risk of death in those receiving prior chemotherapy compared to patients who did not. This detrimental effect of chemotherapy on ICI response and OS could be the results of two factors. First, chemotherapy is associated with immunosuppression characterized by delayed recovery of CD4 $+\mathrm{T}$ cells and B cells [29]. This dampened immune response may abrogate ICI response. Second, it has be shown that chemotherapy elicits gut dysbiosis and poor ICI response, perhaps via gastrointestinal mucositis $[26,30]$. Other medications shown to impact the gut microbiome are PPIs, which may elicit dysbiosis via reduction of intragastric $\mathrm{pH}$ and thus selective pressure for distinct microbial populations [31]. PPI users have been shown to exhibit limited microbial diversity and increased susceptibility to enteric infections [31, 32]. However, in our study we did not find any significant association between PPI use and ICI response or OS. Similarly, another recent study found no effect of PPI use on efficacy of PD-1/PD-L1 blockade in epithelial cancers [33]. Future prospective research studies may provide further insight into the relationship between PPI use, the gut microbiome and ICI response.

\section{Strengths and limitations}

This is a substantially large retrospective, single-institution study examining the effects of prior and concurrent ATBs on clinical response to ICI in patients with NSCLC. Although ATBs were not significantly

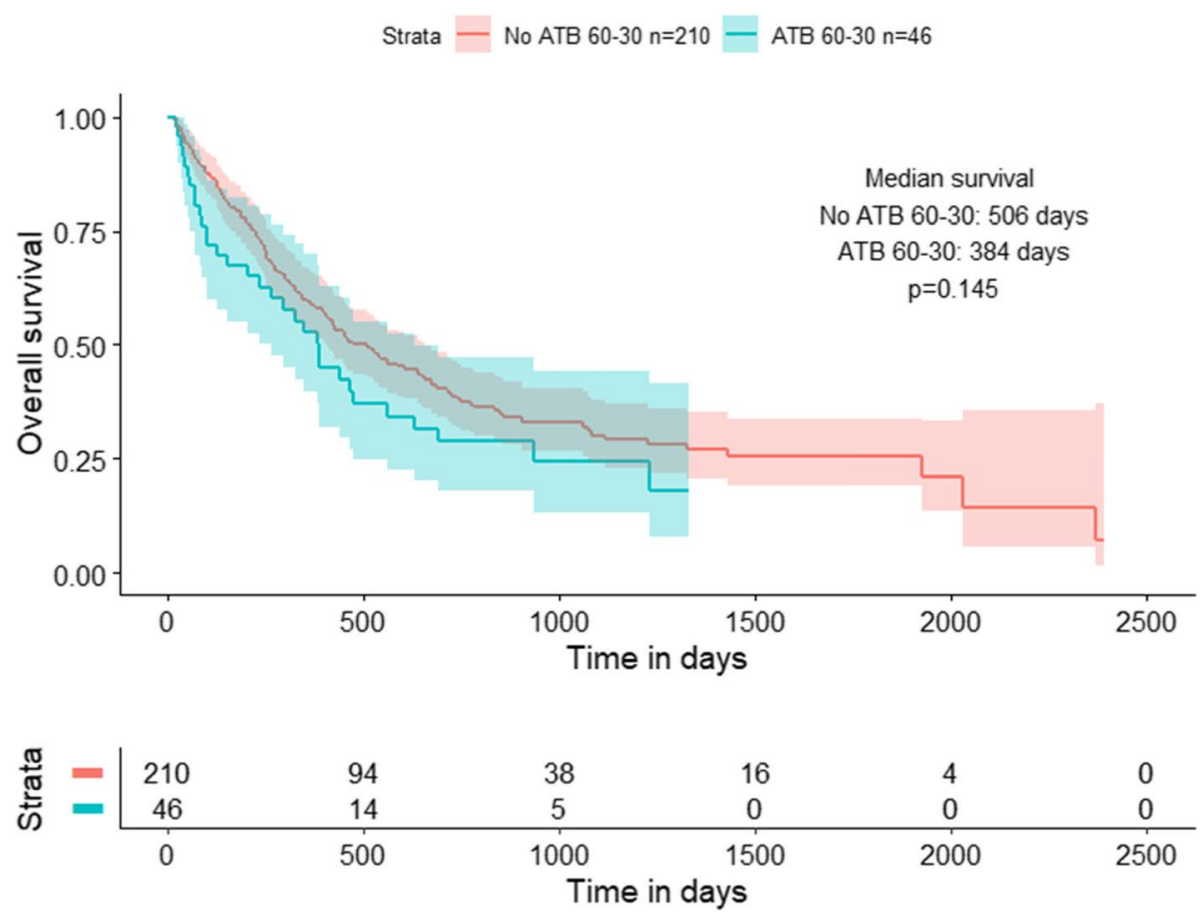

Fig.1 Kaplan Meier survival analysis for the effects of antibiotic use (60-30) on overall survival. Patients receiving antibiotics 60 days before to 30 days after the start of immunotherapy treatment experienced a diminished median survival of approximately 120 days compared to patients without prior exposure to antibiotics within the same time period 


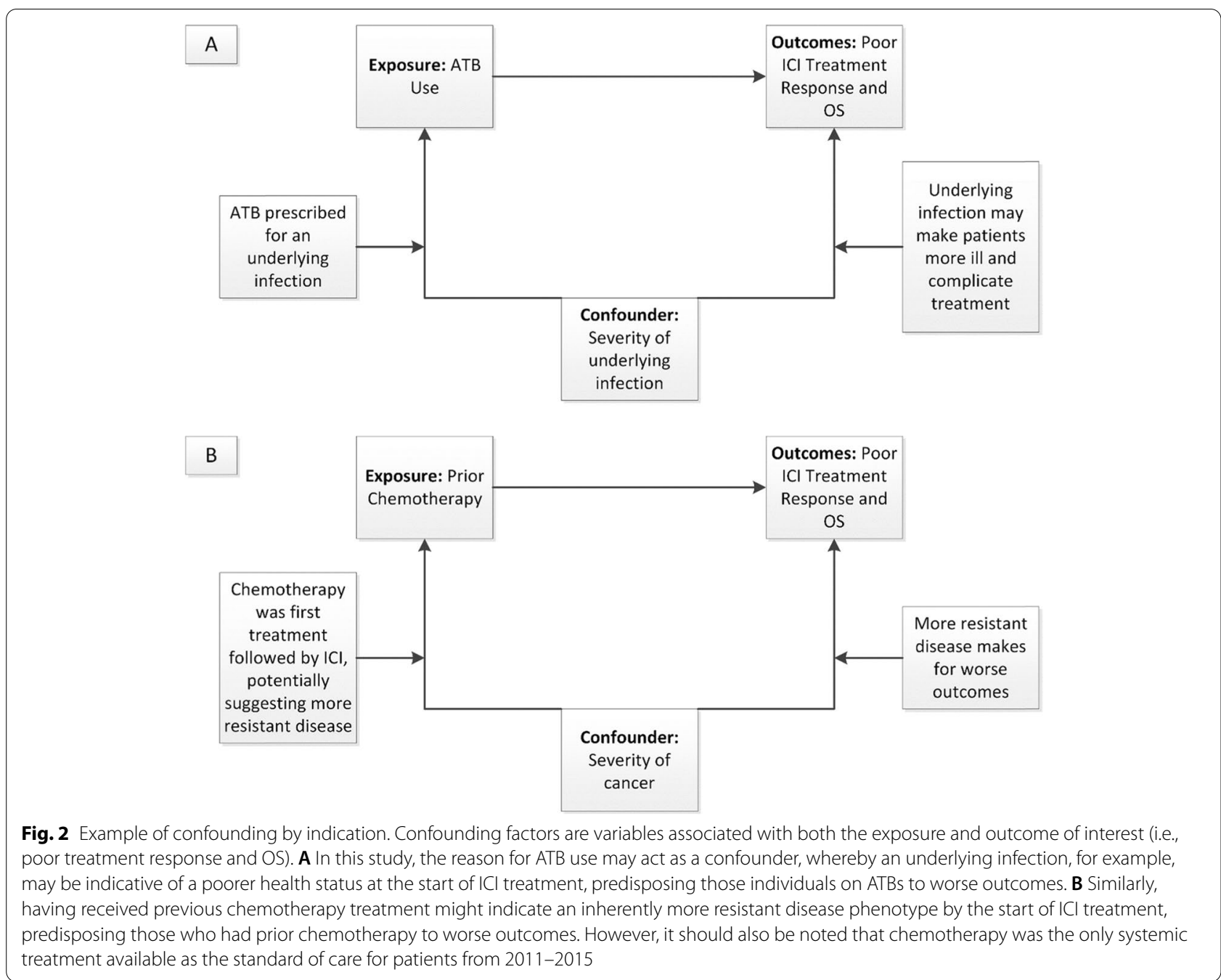

associated with poor clinical outcomes, recent/concurrent ATB use was consistently associated with poorer prognoses.

An important limitation of this retrospective study is the potential impact of confounding by indication [34] (Fig. 2). The association between ATB use and diminished OS identified in this study may be confounded by infectious comorbidity, as has been suggested in other studies of ATB use in patients treated with ICIs [19, 23, $35]$. While $33 \%$ of patients only had one or two doses of prophylactic perioperative ATBs for surgery, the other $67 \%$, who were treated for respiratory infections or for other reasons, may have had poorer health than patients who did not require ATBs and hence, were predisposed to have worse ICI response. Nonetheless, we did not find an association between the reason for ATB use and response, and we controlled for certain co-morbidities in this study using the $\mathrm{CCI}$ and for patient functionality via ECOG performance status, neither of which differed significantly between ATB users and non-users, unlike in previous research studies [36]. It is also possible that concomitant use of corticosteroids and other factors not measured in this study but associated with overall survival in cancer patients, like body mass index, may have confounded the association between ATB use and outcomes [37]. Finally, our study did not document other lifestyle factors that are known to modify the gut microbiome, including diet, exercise, pro- and prebiotics [38], as these were not available retrospectively in the chart review.

Another limitation of this study relates to sample size. Although we retrospectively analyzed a large number of patients over an extended period of time, only 46 received ATBs during the defined time period. Nevertheless, 
exposure misclassification, that is, excluding patients receiving undocumented ATBs from outside healthcare providers, may have influenced the statistical significance of the relationship observed between ATB use and poor ICI outcomes. Also, 33\% of ATB users received only a prophylactic, single dose of ATBs prior to surgery. The effect of a very transient ATB exposure on the gut microbiome is unclear and may have biased results towards the null in this study. Future research should therefore investigate the association between antibiotic use and treatment outcomes in cancer patient cohorts comprised of higher proportions of antibiotic users, preferably in a prospective study for more accurate accounting of antibiotic use.

Although this study addresses ATB use two months before to one month after the start of ICI, it is possible that ATB use during the longer course of immunotherapy treatment may influence clinical outcomes. A recent multicenter study evaluated the influence of both prior and concurrent ATB use on ICI response, but found no significant association between concurrent ATB use and treatment response [23]. Other recent meta-analyses have determined that ATB use within 1-2 months prior to ICI treatment start and within 1-2 months after ICI start resulted in poorer outcomes compared to ATB use at more distant periods of time, highlighting the role of the gut microbiome in priming the immune system and how ATB exposure during an as yet undefined critical priming period may dampen ICI response [19, 20, 35]. Prospective cohort studies are needed to further study associations of concurrent ATB therapy and ICI treatment outcome to better characterize this critical window.

The finding that prior chemotherapy results in worse ICI response and OS is noteworthy. Although the negative effect of chemotherapy in ICI response may be related to the development of dysbiosis of the gut microbiome, there is also the possibility that these chemotherapy patients may have had intrinsically more resistant disease that may not have responded as well to ICI treatment (Fig. 2). However, during the time of the chart review from 2011-2017, ICIs were only available as standard of care off study after FDA approval of nivolumab in 2015 [39], so all stage IV NSCLC patients prior to 2015 routinely received the only available treatment of chemotherapy, which is rarely curative, leading to inevitable relapse. Then, following disease progression, they were treated on study (or off study after 2015) with ICIs. Thus, it is unlikely that more resistant disease accounts for the inferior ICI response observed in this study in patients with prior chemotherapy.

\section{Conclusions}

Our study suggests that patients who receive ATBs near the time of ICI initiation may be more likely to experience poor outcomes than unexposed patients, although differences did not reach statistical significance, warranting further prospective exploration of this association. Prior chemotherapy, on the other hand, resulted in a significant negative impact on ICI treatment response and survival. The current study utilized ATBs and chemotherapy as a proxy for microbial dysbiosis to hypothesize the importance of an eubiotic gut microbiome in mediating immunotherapy efficacy in NSCLC.

Several recent studies suggest that specific compositions of bacteria in the gut microbiome are responsible for driving differential ICI outcomes in patients, although the mechanisms underlying these associations remain largely unknown. In order to investigate whether alterations in the gut microbiome are responsible for mediating ICI efficacy, our research team is beginning a large, prospective study of treatment response and the gut microbiome using metagenomic analyses of prospectively collected stool and blood samples from ICI responders and non-responders, including metabolomics, immune measures, and clinical data to more definitively answer these important questions.

\begin{abstract}
Abbreviations
ATB(s): Antibiotic(s); CCl: Charlson Comorbidity Index; CR: Complete Response; CTLA-4: Cytotoxic T-lymphocyte-Associated Protein 4; ECOG: Eastern Cooperative Oncology Group; FMT: Fecal Microbiota Transfer; ICl: Immune Checkpoint Inhibitor; MCC: Moffitt Cancer Center; NSAIDs: Non-Steroidal Anti-Inflammatory Drugs; NSCLC: Non-Small Cell Lung Cancer; OS: Overall Survival; PD: Progressive Disease; PD-1: Programmed Cell Death-1; PD-L1: Programmed Death Ligand-1; PPIs: Proton-Pump Inhibitor; PR: Partial Response; PS: Performance status; RECIST: Response Evaluation Criteria in Solid Tumors; SD: Stable Disease.
\end{abstract}

\section{Acknowledgements}

We gratefully acknowledge the contributions of Christine M. Pierce and Shirlene Paul, formerly members of the Department of Cancer Epidemiology at Moffitt Cancer Center, to design, data analysis, and manuscript editing.

\section{Author's contributions}

AFN assisted in data abstraction from the patient medical record and was a significant contributor to manuscript writing. SB was a significant contributor to manuscript writing. SH was a significant contributor to manuscript writing. YZ was a significant contributor to data analyses. BM assisted in data abstraction from the patient medical record. SS assisted in data abstraction from the patient medical record. MFG contributed to data abstraction and data analyses. DER contributed to analysis guidance. LAR helped conceptualize the study and was a significant contributor to manuscript writing. All authors read and approved the final manuscript.

\section{Funding}

This research did not receive a specific grant from funding agencies.

\section{Availability of data and materials}

The data in this present study contain confidential patient medical information protected by HIPPA law from public view. It is maintained in a passwordprotected database and not publicly available. The datasets analyzed during the current study are available in a de-identified format from the corresponding author upon reasonable request. 


\section{Declarations}

\section{Ethics approval and consent to participate}

The study protocol was approved by the Chesapeake IRB at 6940 Columbia Gateway Drive, Columbia, MD 21046 (IRB\# 00000790), who determined a waiver of consent given the retrospective design. All methods were performed in accordance with relevant guidelines and regulations. Moffitt Cancer Center's regulatory specialists granted permission for relevant study personnel to review and abstract data from patient records.

\section{Consent for publication}

Not applicable.

\section{Competing interests}

The authors have nothing to report regarding competing interests.

\section{Author details}

'Department of Cancer Epidemiology, Moffitt Cancer Center, Tampa, FL 33612, USA. ${ }^{2}$ Division of Hematology \& Oncology, Moffitt Cancer Center, Tampa, FL 33612, USA. ${ }^{3}$ Department of Thoracic Oncology, Moffitt Cancer Center,

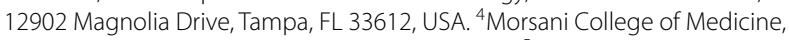
University of South Florida, Tampa, FL 33612, USA. ${ }^{5}$ Cornell University, Ithaca, NY 14853, USA.

Received: 25 May 2021 Accepted: 5 January 2022

Published online: 24 January 2022

\section{References}

1. Ettinger DS, Akerley W, Bepler G, Blum MG, Chang A, Cheney RT, et al. Nonsmall cell lung cancer. JNCCN. 2010;8(7):740-801.

2. Torre LA, Bray F, Siegel RL, Ferlay J, Lortet-Tieulent J, Jemal A. Global cancer statistics, 2012. CA Cancer J Clin. 2015;65(2):87-108.

3. Bareschino MA, Schettino C, Rossi A, Maione P, Sacco PC, Zeppa R, et al. Treatment of advanced non small cell lung cancer. J Thorac Dis. 2011:3(2):122-33.

4. McDonald F, De Waele M, Hendriks LE, Faivre-Finn C, Dingemans AC, Van Schil PE. Management of stage I and II nonsmall cell lung cancer. Eur Respir J. 2017;49:1600764. https://doi.org/10.1183/13993003.00764-2016.

5. Barros JA, Valladares G, Faria AR, Fugita EM, Ruiz AP, Vianna AG, et al. Early diagnosis of lung cancer: the great challenge. Epidemiological variables, clinical variables, staging and treatment. J Bras Pneumol. 2006:32(3):221-7.

6. Kadara H, Kabbout M, Wistuba II. Pulmonary adenocarcinoma: a renewed entity in 2011. Respirology (Carlton, Vic). 2012;17(1):50-65.

7. LuT, Yang X, Huang Y, Zhao M, Li M, Ma K, et al. Trends in the incidence, treatment, and survival of patients with lung cancer in the last four decades. Cancer Manag Res. 2019;11:943-53.

8. Vrankar M, Stanic K. Long-term survival of locally advanced stage III non-small cell lung cancer patients treated with chemoradiotherapy and perspectives for the treatment with immunotherapy. Radiol Oncol. 2018;52(3):281-8.

9. Morgensztern D, Ng SH, Gao F, Govindan R. Trends in stage distribution for patients with non-small cell lung cancer: a National Cancer Database survey. J Thorac Oncol. 2010;5(1):29-33.

10. Gandhi L, Rodríguez-Abreu D, Gadgeel S, Esteban E, Felip E, De Angelis F, et al. Pembrolizumab plus chemotherapy in metastatic non-small-cell lung cancer. N Engl J Med. 2018;378(22):2078-92.

11. Ahmadzadeh M, Johnson LA, Heemskerk B, Wunderlich JR, Dudley $M E$, White $D E$, et al. Tumor antigen-specific CD8 T cells infiltrating the tumor express high levels of PD-1 and are functionally impaired. Blood. 2009;114(8):1537-44.

12. Shui L, Yang X, Li J, Yi C, Sun Q, Zhu H. Gut microbiome as a potential factor for modulating resistance to cancer immunotherapy. Front Immunol. 2019;10:2989.

13. Awad MM, Gadgeel SM, Borghaei H, Patnaik A, Yang JC, Powell SF, et al. Long-Term Overall Survival From KEYNOTE-021 Cohort G: Pemetrexed and Carboplatin With or Without Pembrolizumab as First-Line Therapy for Advanced Nonsquamous NSCLC. J Thorac Oncol. 2021;16(1):162-8.

14. Gopalakrishnan V, Spencer CN, Nezi L, Reuben A, Andrews MC, Karpinets TV, et al. Gut microbiome modulates response to anti-PD-1 immunotherapy in melanoma patients. Sci (New York, NY). 2018;359(6371):97-103.
15. Matson V, Fessler J, Bao R, Chongsuwat T, Zha Y, Alegre ML, et al. The commensal microbiome is associated with anti-PD-1 efficacy in metastatic melanoma patients. Sci (New York, NY). 2018;359(6371):104-8.

16. Routy B, Le Chatelier E, Derosa L, Duong CPM, Alou MT, Daillere R, et al. Gut microbiome influences efficacy of PD-1-based immunotherapy against epithelial tumors. Sci (New York, NY). 2018;359(6371):91-7.

17. Parada Venegas D, De la Fuente MK, Landskron G, González MJ, Quera R, Dijkstra G, et al. Short Chain Fatty Acids (SCFAs)-Mediated Gut Epithelial and Immune Regulation and Its Relevance for Inflammatory Bowel Diseases. Frontiers in Immunology. 2019;10(277). https://doi.org/10.3389/fimmu.2019. 00277.

18. Tsikala-Vafea M, Belani N, Vieira K, Khan H, Farmakiotis D. Use of antibiotics is associated with worse clinical outcomes in patients with cancer treated with immune checkpoint inhibitors: A systematic review and meta-analysis. Int J Infect Dis. 2021;106:142-54.

19. Wilson BE, Routy B, Nagrial A, Chin VT. The effect of antibiotics on clinica outcomes in immune-checkpoint blockade: a systematic review and meta-analysis of observational studies. Cancer Immunol Immunother. 2020;69(3):343-54

20. Huang XZ, Gao P, Song YX, Xu Y, Sun JX, Chen XW, et al. Antibiotic use and the efficacy of immune checkpoint inhibitors in cancer patients: a pooled analysis of 2740 cancer patients. Oncoimmunology. 2019;8(12):e1665973.

21. Pierrard J, Seront E. Impact of the gut microbiome on immune checkpoint inhibitor efficacy-a systematic review. Curr Oncol (Toronto, Ont) 2019:26(6):395-403.

22. Kaderbhai C, Richard C, Fumet JD, Aarnink A, Foucher P, Coudert B, et al. Antibiotic Use Does Not Appear to Influence Response to Nivolumab. Anticancer Res. 2017;37(6):3195-200.

23. Pinato DJ, Howlett S, Ottaviani D, Urus H, Patel A, Mineo T, et al. Antibiotic treatment prior to immune checkpoint inhibitor therapy as a tumor-agnostic predictive correlate of response in routine clinical practice. J Clin Oncol. 2019;37(8_suppl):147.

24 Eisenhauer EA, Therasse P, Bogaerts J, Schwartz LH, Sargent D, Ford R, et al. New response evaluation criteria in solid tumours: revised RECIST guideline (version 1.1). Eur J Cancer. 2009;45(2):228-47.

25. Donoghoe MW, Marschner IC. logbin: An R Package for Relative Risk Regression Using the Log-Binomial Model. J Stat Soft [Internet]. 2018;86(9):1-22.

26. Touchefeu Y, Montassier E, Nieman K, Gastinne T, Potel G, des BrulyVarannes $\mathrm{S}$, et al. Systematic review: the role of the gut microbiota in chemotherapyor radiation-induced gastrointestinal mucositis - current evidence and potential clinical applications. Alimentary Pharmacology and Therapeutics. 2014:40(5):409-21.

27. Sivan A, Corrales L, Hubert N, Williams JB, Aquino-Michaels K, Earley ZM, et al. Commensal Bifidobacterium promotes antitumor immunity and facilitates anti-PD-L1 efficacy. Sci (New York, NY). 2015;350(6264):1084-9.

28. Vetizou M, Pitt JM, Daillere R, Lepage P, Waldschmitt N, Flament C, et al. Anticancer immunotherapy by CTLA-4 blockade relies on the gut microbiota. Sci (New York, NY). 2015;350(6264):1079-84.

29. Verma R, Foster RE, Horgan K, Mounsey K, Nixon H, Smalle N, et al. Lymphocyte depletion and repopulation after chemotherapy for primary breast cancer. Breast Cancer Res. 2016;18(1):10.

30. Montassier E, Gastinne T, Vangay P, des Al-GhalithBruleyVarannes GAS, Massart $\mathrm{S}$, et al. Chemotherapy-driven dysbiosis in the intestinal microbiome. Aliment Pharmacol Ther. 2015;42(5):515-28.

31. Bruno G, Zaccari P, Rocco G, Scalese G, Panetta C, Porowska B, et al. Proton pump inhibitors and dysbiosis: Current knowledge and aspects to be clarified. World J Gastroenterol. 2019;25(22):2706-19.

32. Lo WK, Chan WW. Proton pump inhibitor use and the risk of small intestinal bacterial overgrowth: a meta-analysis. Clin Gastroenterol Hepatol. 2013:11(5):483-90.

33. Mukherjee S, Khalid B, Ibrahimi S, Morton JM, Roman D, Zhao YD, et al. Efficacy of PD-1/PD-L1 therapy: Do proton pump inhibitors affect the outcome? J Clin Oncol. 2018;36(5_suppl):208.

34. Salas M, Hofman A, Stricker BH. Confounding by indication: an example of variation in the use of epidemiologic terminology. Am J Epidemiol. 1999:149(11):981-3.

35. Lurienne L, Cervesi J, Duhalde L, de Gunzburg J, Andremont A, Zalcman G, Buffet R, Bandinelli PA. NSCLC Immunotherapy Efficacy and Antibiotic Use: A Systematic Review and Meta-Analysis. J Thorac Oncol. 2020;15(7):1147-59. https://doi.org/10.1016/j.jtho.2020.03.002 
36. Kim H, Lee JE, Hong SH, Lee MA, Kang JH, Kim IH. The effect of antibiotics on the clinical outcomes of patients with solid cancers undergoing immune checkpoint inhibitor treatment: a retrospective study. BMC Cancer. 2019;19(1):1100

37. Kichenadasse G, Miners JO, Mangoni AA, Rowland A, Hopkins AM, Sorich MJ. Association Between Body Mass Index and Overall Survival With Immune Checkpoint Inhibitor Therapy for Advanced Non-Small Cell Lung Cancer. JAMA Oncol. 2020;6(4):512-8.

38. Hills RD Jr, Pontefract BA, Mishcon HR, Black CA, Sutton SC, Theberge CR Gut Microbiome: Profound Implications for Diet and Disease. Nutrients. 2019;11(7):1613.

39. Borghaei H, Paz-Ares L, Horn L, Spigel DR, Steins M, Ready NE, et al.

Nivolumab versus Docetaxel in Advanced Nonsquamous Non-Small-Cell Lung Cancer. N Engl J Med. 2015;373(17):1627-39.

\section{Publisher's Note}

Springer Nature remains neutral with regard to jurisdictional claims in published maps and institutional affiliations.

- fast, convenient online submission

- thorough peer review by experienced researchers in your field

- rapid publication on acceptance

- support for research data, including large and complex data types

- gold Open Access which fosters wider collaboration and increased citations

- maximum visibility for your research: over 100M website views per year

At BMC, research is always in progress.

Learn more biomedcentral.com/submissions 